\title{
Culture and Sensitivity Patterns of Various Antibiotics Used for the Treatment of Pediatric Infectious Diarrhea in Children under 5 Years of Age: A Tertiary Care Experience from Karachi
}

\author{
Faiza Quraishi' ${ }^{1}$, Shehla Shaheen1, Zahida Memon'1, Ghulam Fatima ${ }^{2}$ \\ ${ }^{1}$ Department of Pharmacology, Ziauddin Medical College, Ziauddin University, Karachi, Pakistan \\ ${ }^{2}$ Department of Microbiology, Central Laboratory, Civil Hospital Karachi, Karachi, Pakistan \\ Email: faiza.quraishi@gmail.com,drshel2011@gmail.com
}

How to cite this paper: Quraishi, F., Shaheen, S., Memon, Z. and Fatima, G. (2018) Culture and Sensitivity Patterns of Various Antibiotics Used for the Treatment of Pediatric Infectious Diarrhea in Children under 5 Years of Age: A Tertiary Care Experience from Karachi. International Journal of Clinical Medicine, 9, 684-696. https://doi.org/10.4236/ijcm.2018.99057

Received: August 6, 2018

Accepted: September 11, 2018

Published: September 14, 2018

Copyright $\odot 2018$ by authors and Scientific Research Publishing Inc. This work is licensed under the Creative Commons Attribution International License (CC BY 4.0).

http://creativecommons.org/licenses/by/4.0/

\section{(c) (i) Open Access}

\begin{abstract}
Background and objective: Infectious diarrhea is one of the most common infections and a significant cause of morbidity and mortality in children under 5 years of age. Frequent and irrational use of antibiotics has resulted in increased bacterial resistance. The aim of our study was to determine the culture and sensitivity patterns of antibiotics used for the treatment of diarrhea in children less than 5 years of age in a tertiary care hospital of Karachi, Pakistan. Methodology: This cross sectional study was conducted for a period of six months in the children of ages ranging between 6 months and 5 years. Stool samples were obtained from the patients presented with signs and symptoms of diarrhea in OPD or being referred to microbiology department for stool C/S (culture and sensitivity). Data were analyzed on SPSS version 19.0. Results: A total number of 325 stool samples were collected, out of which 152 samples were positive for pathogens. The most common pathogen isolated was E. coli 92 (60\%), followed by Klebsiella 56 (37\%) and Salmonella 4 (3\%). All 152 isolates were highly resistant to majority of the standard antibiotics. Conclusion: Infectious diarrhea is highly prevalent among children under 5 years of age. E. coli was found to be the most frequent pathogen isolated in stool samples of the patients presenting with diarrhea and was highly resistant to many of the commonly used standard antibiotics in our clinical set up. Male children were predominantly affected by infectious diarrhea as compared to female children. Therefore, injudicious use of antibiotics should be stopped as well as should not be prescribed empirically for the treatment of all cases of pediatric diarrhea.
\end{abstract}




\section{Keywords}

Infectious Diarrhea, Antibiotic Resistance, Antibiotic Sensitivity, Stool Culture

\section{Introduction}

Universally, diarrhea is one of the most common infectious diseases in humans and is one of the major causes of deaths in children. The most vulnerable age group affected by diarrhea is children less than 5 years of age [1] stands as the second leading cause of death among this age group. WHO defined diarrhea as the passage of three or more loose stools in the last 24 hours [2]. According to WHO updated factsheet (2017), approximately 1.7 billion cases of diarrhea were reported among children, out of which 525,000 children died per year worldwide [3]. In the developed world like Germany, every sixth child under the age of 5 years is affected by diarrhea, once or twice a year [4]. Whereas in under developed world; Africa and South East Asia, mortalities due to diarrhea occur in about $78 \%$ of children. In our neighboring country, India, approximately $14.6 \%$ of children less than 5 years of age group suffered from diarrheal diseases [5].

On the contrary, the scenario related to pediatric diarrheal diseases in our country, Pakistan is grave evident from various local data, out of every 10, one child died before reaching the age of 5 years [6]. In the same year another study revealed that the total population of children less than 5 years of age is $16.34 \%$, out of which $11.76 \%$ suffered from diarrhea [7]. According to Pakistan Demographic and Health Survey conducted in 2013, 53,000 children died due to diarrhea per year [8].

The fundamental principle of treating bacterial diarrhea includes the use of ORS (oral rehydrating solution) along with zinc supplements which helps in reducing volume and duration of the stools [9]. Nevertheless, bacterial diarrhea in children is treated with antibiotics which include ampicillin, cefixime, ceftriaxone, ceftazidime, amikacin, gentamicin, tetracycline, nalidixic acid, ciprofloxacin, co-trimoxazole, etc. [10] [11]. Nowadays, increased antimicrobials' resistance has been reported worldwide. The consequences of increased resistance of antibiotics can cause the prolonged length of the ailment, increased cost of the treatment, manifestations of complications and even death can occur in some children [12].

In Pakistan over-the-counter availability, self-medication as well as irrational and repeated use of antibiotics for the treatment of diarrhea has been observed practiced in our clinical setups. Subsequently due to the overuse of antibiotics, pathogens have attained a high level of resistance and have also resulted in the alteration of the normal gut flora; which could become a risk for future serious infections in children [13] [14]. However, only few studies have been documented in Pakistan regarding the evaluation of the resistance patterns of com- 
monly used antibiotics for diarrhea. Keeping in view all stated facts related to pediatric diarrhea in our community there is an intense urge to conduct more research work with a specific set of goals in order to assess where we are standing in the era of antibiotics' resistance. The aim of this study was to determine the culture and sensitivity patterns of diarrhea in children less than five years of age group in our clinical set up.

\section{Materials and Methods}

This cross-sectional study was conducted in pediatric unit of a tertiary care hospital of Karachi from December 2017 till May 2018, on children of 6 month to 5 years of age. After approval from institutional ethical review committee (ERC) written and informed consents were taken from the patient's parent or guardian. Patient's parents or guardians were instructed to collect at least 5 grams of feces in a given sterilized stool culture bottle. The children included in the study were selected according to the WHO criteria for acute diarrhea i.e. who had at least four liquid stools during the last 24 hours with the signs and symptoms of dehydration. While the children suffering from malabsorption syndrome, systemic infection, severe acute malnutrition, significant medical abnormalities or those who had recently received antibiotic for their current infection were excluded from the study.

According to inclusion criteria, in our study period stool samples were collected from 325 children, out of which 152 samples showed positive bacterial isolates. The stool samples were examined within 4 hours of collection for the presence of bacteria. The samples were grossly and microscopically examined for the color, consistency, presence of cellular elements (RBCs, WBCs, and pus cells), protozoa, eggs and cysts of parasites etc. Fecal specimens for culture were inoculated with the help of swab on SS agar (Salmonella Shigella agar), MacConkeys agar and selenite enrichment broth. The samples were incubated aerobically overnight at 37 degrees Celsius. Next day subculture from selenite F broth on Salmonella shigella agar (SS agar) were also performed. After 24 hours the cultures were re-examined and reported for the presence of organisms i.e. E. coli, klebsiella and salmonella. Serology and biochemical identification were carried out for the confirmation of pathogenic strains, by using; Simon citrate agar, SIM medium agar, Urea agar and TSI agar. All the isolated pathogenic strains were tested for susceptibility to a panel of 16 antibiotics with the Kirby-Bauer disc-diffusion method.

Results were interpreted according to the guidelines of the Clinical Laboratory Standards Institute (CLSI, 2018). According to these guidelines, all the standard antibiotics were tested on 152 stool samples showing positive growths for E. coli, Klebsiella, and Salmonella. After testing these antibiotics the sensitivity and resistance patterns of the bacterial isolates obtained from stool samples were assessed on the basis of zone of inhibitions of respective antibiotics. For the calculation of the zones of inhibition of antibiotics, the diameters of the respective an- 
tibiotic's disc plus the surrounding clear area till the edges of the clear zone (showing no bacterial growth) were measured in millimeters $(\mathrm{mm})$ " with the help of a graduated ruler.

\section{Statistical Analysis}

For data entry and analysis, SPSS version 19.0 was used. Mean and standard deviations were used to express numerical data (Zone of inhibitions and age without grouping), while frequency and percentages were used for age groups, gender, organisms isolated, sensitivity and resistance patterns of antibiotics etc. To find the association between various pediatric age groups, gender and organisms isolated, chi-square test was used. Comparison of average zone of inhibitions of antibiotics with organisms was done by analysis of variance (ANOVA). A p-value of $<0.05$ was considered as statistically significant.

\section{Results}

Most (80\%) of our study population belonged to low socioeconomic background. During our study period, out of 325 stool samples, 152 samples found to be positive. Majority of the stool samples, 92 (60\%) showed E. coli, followed by Klebsiella in 56 (37\%), While only 4 (3\%) samples were positive for Salmonella as presented in Table 1 and Figure 1. The frequency of isolates detected in both genders and in various age subgroups of children less than 5 years age are mentioned in table I, which shows that the frequency of various organisms in both genders and in various age subgroups is different but statistically non-significant $(\mathrm{p}=0.286)$ and $(\mathrm{p}=0.253)$ respectively.

Our results displayed that all 152 isolates were highly resistant to majority of standard antibiotics. Overall, the highest resistance of isolates was shown by ampicillin. Whereas, the antibiotics which has shown least resistance are imipenem and amikacin as given in Table 2.

As shown in Figure 2 and Table 3, the sensitivity pattern of various antibiotics analyzed for individual organism showed that $E$. coli was resistant to majority of commonly used antibiotics, highest resistance was shown for ampicillin (93.5\%), followed by ceftazidime (80\%) and cefotaxime (80\%), whereas most sensitive antibiotic for $E$. coli were found to be amikacin (97.8\%) and imipenem (97.8\%). For klebsiella most resistant antibiotics was ampicillin (92.9\%) and then Amoxicillin-clavulanic acid (82.6\%), while most effective antibiotics were imipenem (96.4\%), amikacin (92.9\%) and gentamycin (92.9\%). While Salmonella was isolated only in 4 samples and maximum sensitivity, (100\%) was observed for amikacin, gentamycin, ciprofloxacin and levofloxacin.

\section{Discussion}

Annually, 1.5 million children lose their lives on account of diarrhea out of the total 10 million deaths in the pediatric age group [3] and it is also regarded as one of the leading causes of morbidity and mortality in children less than 5 years of age throughout the world [15]. 
Table 1. Basic demographic features of children under 5 years of age with diarrhea.

\begin{tabular}{|c|c|c|c|c|c|}
\hline \multirow{2}{*}{\multicolumn{2}{|c|}{ Total stool samples $\mathrm{N}$}} & \multicolumn{3}{|c|}{ Organisms isolated in samples $n=152$} & \\
\hline & & \multirow{2}{*}{$\begin{array}{c}\text { E. coli n (\%) } \\
92(60 \%)\end{array}$} & \multirow{2}{*}{$\begin{array}{c}\text { Klebsiella n (\%) } \\
56(37 \%)\end{array}$} & \multirow{2}{*}{$\begin{array}{c}\begin{array}{c}\text { Salmonella } \\
\text { typhi n (\%) }\end{array} \\
4(3 \%)\end{array}$} & \multirow[b]{3}{*}{ P-value } \\
\hline 3 & & & & & \\
\hline Gender & & & & & \\
\hline Male & 87 & $56(60.9 \%)$ & $30(53.6 \%)$ & $1(25.0 \%)$ & \multirow[b]{2}{*}{0.286} \\
\hline Female & 65 & $36(39.1 \%)$ & $26(46.4 \%)$ & $3(75.0 \%)$ & \\
\hline \multicolumn{6}{|c|}{ Age (months) } \\
\hline $6-12$ & 50 & $32(34.7 \%)$ & $16(28.57 \%)$ & $2(50 \%)$ & \\
\hline $13-24$ & 53 & $35(38.04 \%)$ & $17(30.35 \%)$ & $1(25 \%)$ & \\
\hline $25-36$ & 25 & $16(17.39 \%)$ & $9(16.07 \%)$ & - & 0.253 \\
\hline $37-48$ & 12 & $6(6.52 \%)$ & $6(10.71 \%)$ & - & \\
\hline $49-60$ & 12 & $3(3.26 \%)$ & $8(14.28 \%)$ & $1(25 \%)$ & \\
\hline
\end{tabular}

${ }^{*} \mathrm{P}$ value significance; level: $(\mathrm{P}<0.05)$.

Table 2. Over all antibiotics sensitivity and resistance patterns of bacterial specimens isolated from the stool samples of children with diarrhea, total $\mathrm{N}=152$.

\begin{tabular}{|c|c|c|c|c|}
\hline \multirow{2}{*}{ Antibiotics } & \multirow{2}{*}{$\begin{array}{l}\stackrel{\text { M.I.C. }}{\text { (Minimum inhibitory }} \\
\text { concentration) }(\mu \mathrm{g})\end{array}$} & \multirow{2}{*}{$\begin{array}{c}\text { Z.O.I }(\mathrm{mm}) \\
\begin{array}{c}\text { Mean } \pm \text { std. } \\
\text { deviation }\end{array}\end{array}$} & \multicolumn{2}{|c|}{$\frac{\text { Sensitivity pattern }}{\mathrm{n}(\%)}$} \\
\hline & & & $s$ & $\mathbf{R}$ \\
\hline Ampicillin & 10 & $12.01 \pm 1.82$ & $10(6.5 \%)$ & $142(92.8 \%)$ \\
\hline Amoxicillin & 20 & $11.97 \pm 3.41$ & $22(14.4 \%)$ & $130(85.0 \%)$ \\
\hline Piperacillin/Tazobactam & $100 / 10$ & $21.67 \pm 2.70$ & $134(87.6 \%)$ & $18(11.8 \%)$ \\
\hline Cefotaxime & 30 & $21.90 \pm 3.58$ & $38(24.8 \%)$ & $114(74.5 \%)$ \\
\hline Ceftriaxone & 30 & $20.42 \pm 4.20$ & $50(32.7 \%)$ & $102(66.7 \%)$ \\
\hline Cefuroxime & 30 & $14.17 \pm 4.00$ & $44(28.8 \%)$ & $106(69.3 \%)$ \\
\hline Ceftazidime & 30 & $17.09 \pm 3.61$ & $40(26.1 \%)$ & $112(73.2 \%)$ \\
\hline Cefoperazone/Sulbactam & $75 / 10$ & $19.48 \pm 4.66$ & $108(70.6 \%)$ & $44(28.8 \%)$ \\
\hline Nalidixic acid & 30 & $15.12 \pm 4.62$ & $60(39.2 \%)$ & $92(60.1 \%)$ \\
\hline Ciprofloxacin & 5 & $18.93 \pm 4.82$ & $94(61.4 \%)$ & $58(37.9 \%)$ \\
\hline Levofloxacin & 5 & $15.69 \pm 3.83$ & $92(60.1 \%)$ & $60(39.2 \%)$ \\
\hline Aztreonam & 30 & $17.71 \pm 3.85$ & $54(35.3 \%)$ & $98(64.1 \%)$ \\
\hline Imipenim & 10 & $23.66 \pm 1.77$ & $146(95.4 \%)$ & $6(3.9 \%)$ \\
\hline Amikacin & 30 & $18.75 \pm 1.81$ & $146(95.4 \%)$ & $6(3.9 \%)$ \\
\hline Gentamicin & 10 & $16.50 \pm 1.81$ & $142(92.8 \%)$ & $10(6.5 \%)$ \\
\hline $\begin{array}{c}\text { Trimethoprim/ } \\
\text { Sulfamethoxazole }\end{array}$ & $12.5 / 23.75$ & $11.13 \pm 4.92$ & $54(35.3 \%)$ & $98(64.1 \%)$ \\
\hline
\end{tabular}

Z.O.I = Zone of inhibition (diameter in $\mathrm{mm}$ ), $\mathrm{R}=$ Resistance, $\mathrm{S}=$ Sensitive . 
Table 3. Sensitivity pattern of drug with organisms $(n=152)$.

\begin{tabular}{|c|c|c|c|c|c|c|}
\hline \multirow{3}{*}{ Drugs } & \multicolumn{6}{|c|}{ Organism $(n=152)$} \\
\hline & \multicolumn{2}{|c|}{ E. coli $(\mathrm{n}=92)$} & \multicolumn{2}{|c|}{ Klebsiella $(\mathrm{n}=56)$} & \multicolumn{2}{|c|}{$\begin{array}{l}\text { Salmonella typhi } \\
\quad(\mathrm{n}=4)\end{array}$} \\
\hline & $\begin{array}{c}\text { Sensitive } \\
\mathrm{n}(\%)\end{array}$ & $\begin{array}{c}\text { Resistant } \\
\text { n (\%) }\end{array}$ & $\begin{array}{c}\text { Sensitive } \\
\mathrm{n}(\%)\end{array}$ & $\begin{array}{c}\text { Resistant } \\
\text { n (\%) }\end{array}$ & $\begin{array}{c}\text { Sensitive } \\
\mathrm{n}(\%)\end{array}$ & $\begin{array}{c}\text { Resistant } \\
\mathrm{n}(\%)\end{array}$ \\
\hline Amikacin & $90(97.8)$ & $2(2.2)$ & $52(92.9)$ & $4(7.1)$ & $4(100)$ & - \\
\hline $\begin{array}{l}\text { Amoxicillin- } \\
\text { clavulanic acid }\end{array}$ & $16(17.4)$ & $76(82.6)$ & $6(10.7)$ & $50(89.3)$ & - & - \\
\hline Gentamycin & $86(93.5)$ & $6(6.5)$ & $52(92.9)$ & $4(7.1)$ & $4(100)$ & - \\
\hline Cefuroxime & $32(34.8)$ & $60(65.2)$ & $10(17.9)$ & $46(82.1)$ & $2(50.0)$ & $2(50.0)$ \\
\hline Ceftazidime & $12(13.0)$ & $80(87.0)$ & $28(50.0)$ & $28(50.0)$ & - & - \\
\hline Aztreonam & $28(30.4)$ & $64(69.6)$ & $26(46.4)$ & $30(53.6)$ & - & - \\
\hline Ampicillin & $6(6.5)$ & $86(93.5)$ & $4(7.1)$ & $52(92.9)$ & - & - \\
\hline Cefotaxime & $12(13.0)$ & $80(87.0)$ & $26(46.4)$ & $30(53.6)$ & - & - \\
\hline Ciprofloxacin & $54(58.7)$ & $38(41.3)$ & $36(64.3)$ & $20(35.7)$ & $4(100)$ & - \\
\hline Nalidixic acid & $24(26.1)$ & $68(73.9)$ & $36(64.3)$ & $20(35.7)$ & - & - \\
\hline Levofloxacin & $50(54.3)$ & $42(45.7)$ & $38(67.9)$ & $18(32.1)$ & $4(100)$ & - \\
\hline Ceftriaxone & $34(37.0)$ & $58(63.0)$ & $14(25.0)$ & $42(75.0)$ & $2(50.0)$ & $2(50.0)$ \\
\hline Imipenem & $90(97.8)$ & $2(2.2)$ & $54(96.4)$ & $2(3.6)$ & $2(50.0)$ & $2(50.0)$ \\
\hline $\begin{array}{l}\text { Sulfamethoxazole } \\
\text { Trimethoprim }\end{array}$ & $32(34.8)$ & $60(65.2)$ & $20(35.7)$ & $36(64.3)$ & $2(50.0)$ & $2(50.0)$ \\
\hline $\begin{array}{l}\text { Cefoperazone/ } \\
\text { Sulbactam }\end{array}$ & $58(63.0)$ & $34(37.0)$ & $46(82.1)$ & $10(17.9)$ & $4(100)$ & - \\
\hline $\begin{array}{l}\text { Piperacillin/ } \\
\text { Tazobactam }\end{array}$ & $78(84.9)$ & $14(15.1)$ & $52(92.9)$ & $4(7.1)$ & $4(100)$ & - \\
\hline
\end{tabular}

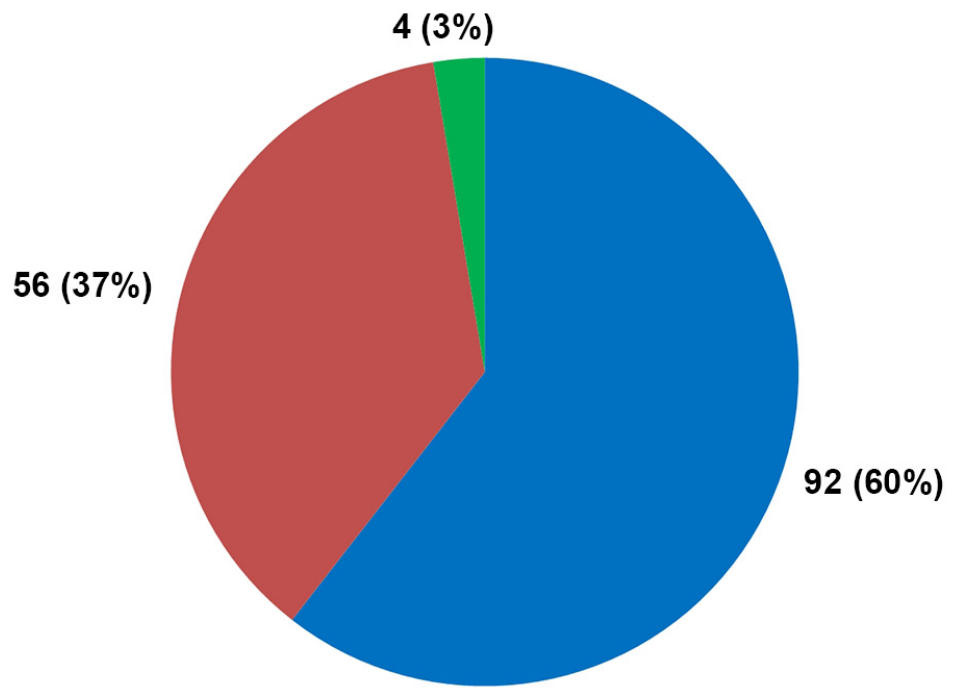

$\square$ E. coli $₫$ Klebsiella $\square$ Salmonella Typhi

Figure 1. Distribution of organisms. 


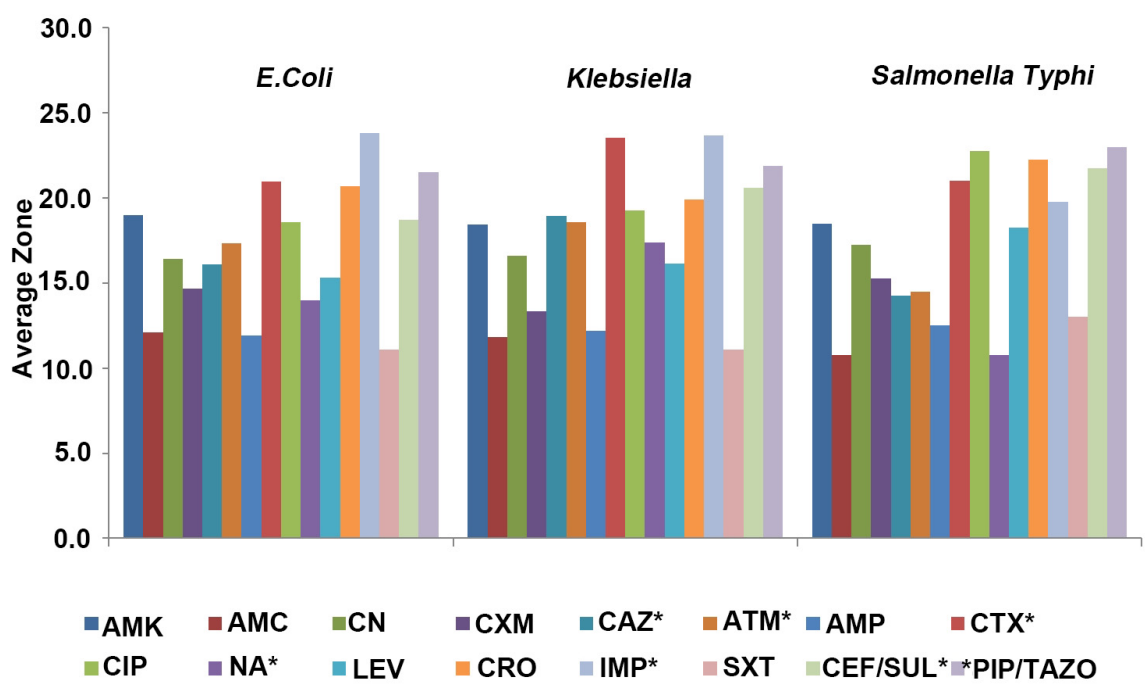

Figure 2. Average Zone of inhibitions of different antibiotics with organisms. AMK; Amikacin, AMC; Amoxicillin-clavulanic acid, CN; Gentamycin, CXM; Cefuroxime, CAZ; Ceftazidime, ATM; Aztreonam, AMP; ampicillin, CTX; cefotaxime, CIP; Ciprofloxacin, NA; Nalidixic acid, LEV; Levofloxacin, CRO; Ceftriaxone, IMP; Imipenem, SXT; Trimethoprim-Sulfamethoxazole, CEF/SUL; Cefoperazone/Sulbactam, PIP/TAZO; Piperacillin/Tazobactam [ ${ }^{*}$ shows significant $\mathrm{P}$ values].

Pakistan is one of the fifteen developing countries, which has shown the highest burden of diarrheal diseases [16]. Our study conducted at pediatric unit of a tertiary care hospital of Karachi displayed that most (80\%) of the cases of pediatric diarrhea were presented from low socioeconomic class. According to our study, 152 (46.77\%) stool samples were found to be positive for bacterial pathogens out of 325 total samples as shown in table I, which revealed that infectious diarrhea is highly prevalent in our clinical set up. One of the previous studies conducted from the year 2002 till 2006 on children $<5$ years of age living in squatter settlements of central Karachi, displayed that the burden of infectious diarrhea varied from $29 \%$ to $37 \%$ [17]. In 2016, WHO statistics of Pakistan stated the prevalence of diarrhea in children is $23 \%$ [18]. This is clearly indicating that disease burden of diarrhea is rising in our population with time. Presumably the reason behind rising pattern and higher burden of diarrheal disease among pediatric age group in our country is that the major chunk of our study population comes from the low socioeconomic areas which are directly linked to poor quality of drinking water and hygiene especially hand washing, improper disposal of waste material, lack of health education of mothers, immunization and nutritional status not maintained according to the age. This is quite evident from a report published in 2017 in the most leading and widely read English newspaper Dawn, which highlighted that Pakistan is the seventh worst country in terms of access to basic sanitation, as its 42 percent of the population remains without access to basic sanitation [19]. On the basis of National Nutritional Survey conducted in different parts of the world the frequency of diarrhea in children less than 5 years of age was found to be variable, ranging from $22.3 \%$ in 
Ethiopia, 22.1\% in Iraq, 21.3\% in Egypt and 12\% in Tanzania. In 2010, the study performed in Fatemieh children hospital, Central Iran, 514 (57.6\%) children were diagnosed with infectious gastroenteritis [18].

According to our results more bacterial isolates were obtained from stool samples of male children i.e. $57 \%$ as compared to female children in which $43 \%$, of samples were positive (Table 1 ) but this difference was non-significant ( $\mathrm{p}=$ 0.286 ) as shown in table I. Whereas, the study published in the journal of Global Health Action in 2014, revealed that there was no gender predominance related to the frequency of diarrhea among children less than 5 years of age [20]. Another study conducted in central Iran in 2010, also showed insignificant difference in the frequency of infectious diarrhea between male and female children $(\mathrm{p}=0.22)[21]$.

In our study, $E$. coli was the most common pathogen isolated in 92 (60\%) stool samples out of the total 152, followed by the second common organism Klebsiella $56(37 \%)$ and then Salmonella $4(3 \%)$ as shown in Figure 1. E. coli is the facultative anaerobic gram negative bacillus which belongs to the family of Enterobacteriaceae. It is transmitted through fecal-oral route by using contaminated water and food. It was also detected in the milk given to the infants and young children through feeding bottles which were mishandled by the uneducated mothers especially seen in the children coming from low socioeconomic background [22]. Similarly, E. coli was detected to be the frequent cause of acute infectious diarrhea in the developing countries, Sudan and India [10] [23]. On the contrary recent studies conducted in Turkey and Spain, displayed the highest number of Campylobacter and Salmonella species isolated from stool samples of children having diarrhea [24] [25]. Likewise, in the region of South America, Ecuador, Shigella species and Campylobacter jejuni were found to be prevalent, whereas in China, Shigella species were found to be the most common agents for pediatric infectious diarrhea [26] [27].

Our current study showed that the majority of cases of E. coli and klebsiella were found in 13 - 24 months of age, subsequently the number of cases decreased in 49 - 60 and 37 - 48 months of age respectively as shown in table I. Similar results were presented by Amir Saeed et al. in 2015, which stated, E. coli is the most common causative agent of diarrhea in children under five year of age in Khartoum, Sudan [10]. Another study published in Tanzania 2014, the epidemics of diarrhea is significantly higher in the age groups of 18 - 23 months [28].

Various microorganisms are resistant to multiple antibiotics and hence named as multidrug resistant organisms. In our study the organism which showed the resistance to majority (more than $60 \%$ ) of the antibiotics is E. coli. The study published in the American Journal of Tropical Medicine and Hygiene stated that Escherichia coli (E. coli) is the most frequently occurring organism in children under 5 years of age and is almost entirely resistant to many antibiotics [29]. As mentioned in Figure 2 and Table 3, our current study revealed that E. coli is re- 
sistant to majority of the commonly prescribed antibiotics, appropriate for pediatric age group, including among penicillins (amoxiclav, ampicillin, amoxicillin), cephalosporins (cefotaxime, ceftriaxone cefuroxime, ceftazidime), while gentamicin, aztreonam, and trimethoprim-sulfmethoxazole were also resistant. Likewise, Klebsiella has also showed resistance against commonly prescribed antibiotics, amoxicillin, cefuroxime, cefotaxime, ceftriaxone, ampicillin and trimethoprim-sulfmethoxazole. Although in our study, Salmonella was isolated in 4 samples only out of the total 152 but like other two organisms Salmonella has also shown higher resistance for cephalosporins (cefuroxime, ceftriaxone) and trimethoprim-sulfmethoxazole. Surprisingly in comparison to both of the organisms, Salmonella has showed higher resistance to imipenem as well, which is not prescribed routinely and is reserved for infections due to resistant Pseudomonas Aeruginosa [30]. Interestingly the intra group comparison of sensitivity of E. coli, Klebsiella and Salmonella displayed that among all antibiotics, the ZOIs of ceftazidime, aztreonam, ceftriaxone, nalidixic acid, imipenem and cefoperazone/sulbactam were highly significant for all the three organisms as shown in Figure 2, probably showing the clear difference between ZOIs of sensitive and resistant antibiotics mentioned above for all of the three organisms. Similarly, a recent study published in Feb. 2018, Italy, precisely exposed E. coli strains resistant to ampicillin, cotrimoxazole, chloramphenicol, ceftriaxone, and ceftazidime. In the same study, Klebsiella species were found resistant to ampicillin, cefotaxime, cefuroxime, co-amoxiclav, mezlocillin, chloramphenicol, gentamicin, and ceftazidime, whereas Salmonella strains were resistant to ampicillin, cephalotin, ceftriaxone, gentamicin, amikacin, trimethoprim-sulfamethoxazole, chloramphenicol, and tetracycline [31]. Despite that Italy is among developed countries and our country, Pakistan is a developing country but the scenario of antibiotics resistance is same in both of the countries, possibly this is due to empirical and extensive use of antibiotics in clinical set-ups of both countries. Our results were also parallel to studies reported in other countries like China, which also approximately demonstrates the comparative outcomes [32].

In our study most sensitive antibiotic for E. coli and Klebsiella were shown to be aminoglycosides (amikacin and gentamycin) as well as imipenem, while Salmonella showed highest sensitivity (100\%) to both aminoglycosides and fluoroquinolones (ciprofloxacin and levofloxacin) but was resistant to imipenem (50\%) as shown in Figure 2 and Table 3. However, the study conducted in India 2016, showed the highest sensitive (100\%) antibiotics for E. coli and Klebsiella are ciprofloxacin, norfloxacin and gentamicin [33]. Whereas, the data as observed in Ethiopia 2017 reveals, Salmonella is highly sensitive to ceftriaxone and norfloxacin but resistant to amoxicillin [34].

In the light of above discussion it is evident that currently majority of commonly prescribed antibiotics, suitable for pediatric use in the treatment of bacterial diarrhea have been resistant. The reason is that globally the empirical use of antibiotics for different clinical situations as well as for all cases of pediatric 
diarrhea has been increased. Furthermore in our country, over-the-counter availability of various antibiotics has led people to self-medications. Subsequently this has increased resistance of highly useful antibiotics in our population, hence limited antibiotics are left for the treatment of serious infections in pediatric age group as majority of the sensitive drugs, such as aminoglycosides, fluoroquinolones and imipenem cannot be used in children due to systemic toxic effects.

\section{Conclusion}

Infectious diarrhea is highly prevalent among children of less than 5 years of age, affecting more commonly male children in our clinical setup. E. coli is responsible for majority of the cases and was found to be highly resistant to many of the standard antibiotics used currently for the treatment of infectious diarrhea in children. Although in comparison, our study displayed that imipenem, fluoroquinolones and gentamycin have better sensitivity profiles but owing to their limited use in pediatric age group, could not be recommended in pediatric diarrhea leaving less treatment options for this age group. This situation is quite grave and can lead to even greater number of morbidity and mortality in this age group on account of diarrhea only.

\section{Recommendations}

In order to minimize the resistance, injudicious use of antibiotics should be stopped as well as should not be prescribed empirically for the treatment of all cases of pediatric diarrhea. The decision of giving antibiotics should be based on patient's detailed history, clinical examination and according to the culture and sensitivity reports. Subsequently, this will help in reducing the cost and economic burden due to the overuse of antibiotics on our underprivileged population and will also improve the overall health of children.

The increasing resistance of antibiotics in children requires serious measures and implementations at national and international levels which emphasizes the need to explore other simple, supportive, effective and alternative treatment for resolving the burden of diarrheal diseases in children less than 5 years of age. Furthermore, the use of some antibiotics should be restricted to serious systemic and life-threatening infections in pediatric age group.

\section{Conflicts of Interest}

The authors declare no conflicts of interest regarding the publication of this paper.

\section{References}

[1] Zheng, S., Yu, F., Chen, X., Cui, D., Cheng, Y., Xie, G., Chen, Y., et al. (2016) Enteropathogens in Children Less than 5 Years of Age with Acute Diarrhea: A 5-Year Surveillance Study in the Southeast Coast of China. BMC Infectious Diseases, 16, 434. https://doi.org/10.1186/s12879-016-1760-3 
[2] Pawlowski, S.W., Warren, C.A. and Guerrant, R. (2009) Diagnosis and Treatment of Acute or Persistent Diarrhea. Gastroenterology, 136, 1874-1886. https://doi.org/10.1053/j.gastro.2009.02.072

[3] http://www.who.int/en/news-room/fact-sheets/detail/diarrhoeal-disease

[4] Van Damme, P., Giaquinto, C., Huet, F., Gothefors, L., Maxwell, M., Van der Wielen, M. and REVEAL Study Group (2007) Multicenter Prospective Study of the Burden of Rotavirus Acute Gastroenteritis in Europe, 2004-2005: The REVEAL Study. The Journal of Infectious Diseases, 195, S4-S16. https://doi.org/10.1086/516714

[5] Singh, A. and Singh, M.N. (2014) Diarrhoea and Acute Respiratory Infections among Under-Five Children in Slums: Evidence from India (No. e208v1). Peer), Preprints.

[6] https://dailytimes.com.pk/229126/breakthrough-diarrhoea-treatment-launched-inpakistan/

[7] Murtaza, F., Mustafa, T. and Awan, R. (2015) Child Health Inequalities and Its Dimensions in Pakistan. Journal of Family \& Community Medicine, 22, 169.

https://doi.org/10.4103/2230-8229.163036

[8] https://www.thenews.com.pk/print/67968-53000-children-die-in-pakistan-due-to-d iarrhoea

[9] Cruchet, S., Furnes, R., Maruy, A., Hebel, E., Palacios, J., Medina, F., Xóchihua, L., et al. (2015). The Use of Probiotics in Pediatric Gastroenterology: A Review of the Literature and Recommendations by Latin-American Experts. Pediatric Drugs, 17, 199-216. https://doi.org/10.1007/s40272-015-0124-6

[10] Saeed, A., Abd, H. and Sandstrom, G. (2015) Microbial Aetiology of Acute Diarrhoea in Children under Five Years of Age in Khartoum, Sudan. Journal of Medical Microbiology, 64, 432-437. https://doi.org/10.1099/jmm.0.000043

[11] Diniz-Santos, D.R., Silva, L.R. and Silva, N. (2006) Antibiotics for the Empirical Treatment of Acute Infectious Diarrhea in Children. Brazilian Journal of Infectious Diseases, 10, 217-227. https://doi.org/10.1590/S1413-86702006000300011

[12] Bruzzese, E., Giannattasio, A. and Guarino, A. (2018) Antibiotic Treatment of Acute Gastroenteritis in Children. F1000Research, London, 7.

[13] Irshad, R., Bibi, S., Ahmed, W., Alam, S.E. and Hasan, F. (2017) Synergistic Effect of Antibiotics against Bacterial Pathogens Causing Diarrhea Isolated from Children < 5 Years of Age. Pakistan Journal of Medical Research, 56, 131-134.

[14] Hameed, A., Naveed, S., Qamar, F., Alam, T. and Abbas, S.S. (2016) Irrational Use of Antibiotics. Different Age Groups of Karachi: A Wakeup Call for Antibiotic Resistance and Future Infections. Journal of Bioequivalence \& Bioavailability, 8, 242-245.

[15] Jones, S. (2003) A Clinical Pathway for Pediatric Gastroenteritis. Gastroenterology Nursing, 26, 7-18. https://doi.org/10.1097/00001610-200301000-00003

[16] https://www.dawn.com/news/1077426

[17] Luby, S.P., Agboatwalla, M. and Hoekstra, R.M. (2011) The Variability of Childhood Diarrhea in Karachi, Pakistan, 2002-2006. The American Journal of Tropical Medicine and Hygiene, 84, 870-877. https://doi.org/10.4269/ajtmh.2011.10-0364

[18] Asim, M., Nawaz, Y., Sohail, M.M., Khalid, I. and Ain, Q. (2017) Childhood Illness Prevalence and Health Seeking Behavior of Mothers in Faisalabad, Pakistan. Rawal Medical Journal, 42, 563-566. 
[19] DAWN Newspaper Lahore. https://www.dawn.com/news/1372293

[20] Diouf, K., Tabatabai, P., Rudolph, J. and Marx, M. (2014) Diarrhoea Prevalence in Children under Five Years of Age in Rural Burundi: An Assessment of Social and Behavioural Factors at the Household Level. Global Health Action, 7, Article ID: 24895. https://doi.org/10.3402/gha.v7.24895

[21] Habibinejad, H.A., Riahin, A.A., Heidari, A. and Mahjourian, F. (2010) Infectious Diseases in Hospitalized Children of Central Iran. Pakistan Journal of Medical Sciences, 26, 901-904.

[22] Gomes, T.A., Elias, W.P., Scaletsky, I.C., Guth, B.E., Rodrigues, J.F., Piazza, R.M., Martinez, M.B., et al. (2016) Diarrheagenic Escherichia coli. Brazilian Journal of Microbiology, 47, 3-30. https://doi.org/10.1016/j.bjm.2016.10.015

[23] Rathaur, V.K., Pathania, M., Jayara, A. and Yadav, N. (2014) Clinical Study of Acute Childhood Diarrhoea Caused by Bacterial Enteropathogens. Journal of Clinical and Diagnostic Research, 8, PC01.

[24] Sánchez-Capilla, A.D., Sorlózano-Puerto, A., Rodríguez-Granger, J., Martínez Brocal, A., Navarro-Marí, J.M. and Gutiérrez-Fernández, J. (2015) Infectious Etiology of Diarrheas Studied in a Third-Level Hospital during a Five-Year Period. Revista Española de Enfermedades Digestivas, 107, 89-97.

[25] Bicer, S., Col, D., Erdag, G.C., Giray, T., Gurol, Y., Yilmaz, G., Ozelgun, B., et al. (2014) A Retrospective Analysis of Acute Gastroenteritis Agents in Children Admitted to a University Hospital Pediatric Emergency Unit. Jundishapur Journal of Microbiology, 7, e9148.

[26] Vasco, G., Trueba, G., Atherton, R., Calvopina, M., Cevallos, W., Andrade, T., Eisenberg, J.N., et al. (2014) Identifying Etiological Agents Causing Diarrhea in Low Income Ecuadorian Communities. The American Journal of Tropical Medicine and Hygiene, 91, 563-569. https://doi.org/10.4269/ajtmh.13-0744

[27] Wang, X., Wang, J., Sun, H., Xia, S., Duan, R., Liang, J., Jing, H., et al. (2015) Etiology of Childhood Infectious Diarrhea in a Developed Region of China: Compared to Childhood Diarrhea in a Developing Region and Adult Diarrhea in a Developed Region. PLoS ONE, 10, e0142136. https://doi.org/10.1371/journal.pone.0142136

[28] Mashoto, K.O., Malebo, H.M., Msisiri, E. and Peter, E. (2014) Prevalence, One Week Incidence and Knowledge on Causes of Diarrhea: Household Survey of Under-Fives and Adults in Mkuranga District, Tanzania. BMC Public Health, 14, 985. https://doi.org/10.1186/1471-2458-14-985

[29] Al-Gallas, N., Bahri, O., Bouratbeen, A., Haasen, A.B. and Aissa, R.B. (2007) Etiology of Acute Diarrhea in Children and Adults in Tunis, Tunisia, with Emphasis on Diarrheagenic Escherichia coli: Prevalence, Phenotyping, and Molecular Epidemiology. The American Journal of Tropical Medicine and Hygiene, 77, 571-582. https://doi.org/10.4269/ajtmh.2007.77.571

[30] Ameen, N., Memon, Z., Shaheen, S., Fatima, G. and Ahmed, F. (2015) Imipenem Resistant Pseudomonas aeruginosa: The Fall of the Final Quarterback. Pakistan Journal of Medical Sciences, 31, 561.

[31] Pacifici, G.M. (2018) Multidrug Resistance in Infants and Children. International Journal of Pediatrics, 6, 7055-7080.

[32] Zhou, Y., Zhu, X., Hou, H., Lu, Y., Yu, J., Mao, L. and Sun, Z. (2018) Characteristics of Diarrheagenic Escherichia coli among Children under 5 Years of Age with Acute Diarrhea: A Hospital Based Study. BMC Infectious Diseases, 18, 63. https://doi.org/10.1186/s12879-017-2936-1 
[33] Srinivasarao, J., Chinnoutpalli, D.V. and Sarada, D. (2016) Bacteriological Study of Diarrheas in Children. International Journal of Current Microbiology and Applied Sciences, 5, 778-783. https://doi.org/10.20546/ijcmas.2016.506.086

[34] Terfassa, A. and Jida, M. (2018) Prevalence and Antibiotics Susceptibility Pattern of Salmonella and Shigella Species among Diarrheal Patients Attending Nekemte Referral Hospital, Oromia, Ethiopia. International Journal of Microbiology, 2018, Article ID: 9214689. 